\title{
Overview of co-deposition and fuel inventory in castellated divertor structures at JET
}

\author{
M.J. Rubel ${ }^{\mathrm{a}, *}$, J.P. Coad ${ }^{\mathrm{b}}$, R.A. Pitts ${ }^{\mathrm{c}}$, \\ Contributors to the JET-EFDA Workprogramme ${ }^{1}$ \\ ${ }^{a}$ Alfvén Laboratory, Royal Institute of Technology (KTH), Association EURATOM-VR, 10044 Stockholm, Sweden \\ b Culham Science Centre, EURATOM-UKAEA Fusion Association, Abingdon, Oxon OX14 3DB, United Kingdom \\ ${ }^{\mathrm{c}}$ Centre de Recherches en Physique des Plasmas, Association EURATOM - Conféderation Suisse, \\ École Polytechnique Fédérale de Lausanne, 1015 Lausanne, Switzerland
}

\begin{abstract}
The main focus of this work is fuel retention in plasma components of the JET water-cooled Mk-I divertors operated with small tiles, first with carbon fibre composite (CFC) and then with castellated beryllium. Until recently these have been the only large-scale structures of this type used in fusion experiments. Three issues regarding fuel retention and material migration are addressed: (i) accumulation in gaps separating tiles and in the grooves of castellation; (ii) comparison of deposition on carbon and beryllium; (iii) in-depth migration of deuterium into the bulk of CFC. The essential results are summarised as follows: (i) co-deposition occurs up to a few cm deep in the gaps between the Mk-I tiles; (ii) fuel inventory in the CFC tile gaps exceeds that on plasma-facing surfaces by up to a factor of 2; (iii) in gaps between the beryllium tiles from the inner divertor corner the fuel content reaches $30 \%$ of that on plasma-facing surfaces, whereas in the grooves of castellation in Be the fuel content is less than 3.0\% of that found on the top surface; (iv) fuel inventory on the Be tiles is strongly associated with the carbon co-deposition; (v) the D content measured in the bulk (1.5 mm below the surface) on cleaved CFC tiles exceeds $1 \times 10^{15} \mathrm{~cm}^{-2}$. Implications of these results for a next-step device are addressed and the transport mechanism into the gaps is briefly discussed. The results presented here suggest that in a machine with non-carbon walls in the main chamber (as foreseen for ITER) the material transport and subsequent fuel inventory in the castellation would be reduced.
\end{abstract}

(C) 2007 Elsevier B.V. All rights reserved.

\footnotetext{
* Corresponding author.

E-mail addresses: rubel@kth.se, marek.rubel@alfvenlab. kth.se (M.J. Rubel).

${ }^{1}$ See the Appendix to the paper of J. Pamela et al., Fusion Energy 2004 (Proceedings of the 20th International Fusion Energy Conference, Vilamoura, 2004), IAEA, Vienna (2004).
}

\section{Introduction}

Safety and economy of operation of a future fusion reactor are the driving forces for the assessment of in-vessel fuel accumulation. The issue of tritium inventory has long been recognised but its full importance was realised only after the D-T campaigns performed in tokamaks with a carbon-based 
first wall: JET [1] and TFTR [2]. The divertor geometry and related power deposition profiles have a strong impact on the transport of eroded material and its co-deposition with the fuel species [3]. For this reason, an important mission of the JET tokamak is to optimise the wall and divertor geometry and to test a variety of plasma operation scenarios in order to provide the best possible predictions for ITER.

Over the years JET has been operated with a series of divertors including the Mk-I structure in which first carbon fibre composites (CFC), and then beryllium tiles were used. Beryllium has also been regularly used at JET for coating of main chamber carbon plasma-facing components (PFCs) with an evaporated Be layer. When fuel retention and material transport are considered, the simultaneous use of carbon and beryllium is very important for at least two reasons: (i) ITER plans to use both of these materials; (ii) it helps to distinguish the influence chemical and physical processes in wall erosion and to draw conclusions regarding their impact on fuel inventory.

Following the full D-T campaign at JET with the Mk-IIA divertor structure, the most pronounced fuel accumulation was found in the inner divertor corner, in particular, in remote areas shadowed from direct plasma line-of-sight $[3,4]$. As a result of these observations, detailed studies have been undertaken to assess the material fuel retention and transport to shadowed regions of other JET divertors [4,5]. Emphasis has been placed on studies of re-deposition and fuel retention in certain construction features similar to those foreseen for ITER, e.g. castellated tiles. Such a tile structure is thought to be the best solution to ensure the thermo-mechanical durability and integrity of materials under high heat flux loads, especially when considering the use of metals (W and Be) [6]. Although deposition in gaps between PFC tiles has been studied in several machines [7-9], little is known about the fuel retention in narrow grooves. Until recently, the JET Mk-I divertor had been the only large-scale castellated structure used in fusion experiments. Analysis of deposition in the narrow castellated grooves and in tile gaps of the Mk-I structure can therefore contribute to the assessment of material migration with the added, and important benefit, of an environment containing both $\mathrm{C}$ and Be. The general issue of material migration into gaps and the subsequent fuel retention are of crucial importance for ITER, in which there will be many such areas. All of them will be difficult to access by cleaning methods for fuel removal.

The specific objectives of the work presented here are: (i) to provide an overview of fuel inventory in the gaps separating tiles and in the grooves of castellation; (ii) to assess the impact of the divertor material on the fuel retention in such areas; (iii) to determine the in-depth migration of deuterium into the bulk of CFC used at the JET divertor. The latter point is a continuation of studies of long-term fuel retention in the bulk of carbon-based composites exposed to hydrogen isotopes in tokamaks and laboratory plasma facilities $[10,11]$.

\section{Experimental}

The Mk-I divertor was a water-cooled (base temperature $30-50{ }^{\circ} \mathrm{C}$ ) structure composed of small roof-shaped tiles: $72 \mathrm{~mm}$ long poloidally, $30-40 \mathrm{~mm}$ wide toroidally, $50 \mathrm{~mm}$ high and separated by gaps either 6 or $10 \mathrm{~mm}$ wide. As a result of roof-shaping and arrangement of the tiles with respect to the magnetic field lines, the lower part of a given tile was shadowed by the upper edge of the adjacent neighbour. Views of the JET vessel with the Mk-I divertor and the arrangement of tiles with respect to the magnetic field lines can be found elsewhere $[9,12]$. The divertor was first operated for $\sim 60000 \mathrm{~s}$ with CFC tiles and then for $\sim 20000 \mathrm{~s}$ with castellated $(6 \times 6 \mathrm{~mm}$ with $6 \mathrm{~mm}$ deep and $0.6 \mathrm{~mm}$ wide groove) beryllium blocks. Fig. 1 illustrates the Mk-I divertor components with castellation grooves and gaps between the beryllium tiles.

Tiles retrieved from the torus were examined $e x$ situ by means of nuclear reaction analysis (NRA) with a ${ }^{3} \mathrm{He}^{+}$beam to quantify and map surface contents of deuterium, beryllium and carbon. Most of the $\mathrm{D}, \mathrm{Be}$ and $\mathrm{C}$ analyses were carried out with a $2.5 \mathrm{MeV}^{3} \mathrm{He}^{+}$beam, but to assess the thickness of deuterium-containing deposits, the ${ }^{3} \mathrm{He}$ beam energy was occasionally scanned in the range $0.7-$ $3 \mathrm{MeV}$ varying the information depth from $1.3 \mu \mathrm{m}$ to approximately $10 \mu \mathrm{m}$, respectively. This highest attainable information depth was still too small in case of the thickest deposits (approx. $25 \mu \mathrm{m}$ ).

\section{Results and discussion}

\subsection{Mk-I divertor with CFC tiles}

Following long-term operation there was a significant deposition on both the plasma-facing 


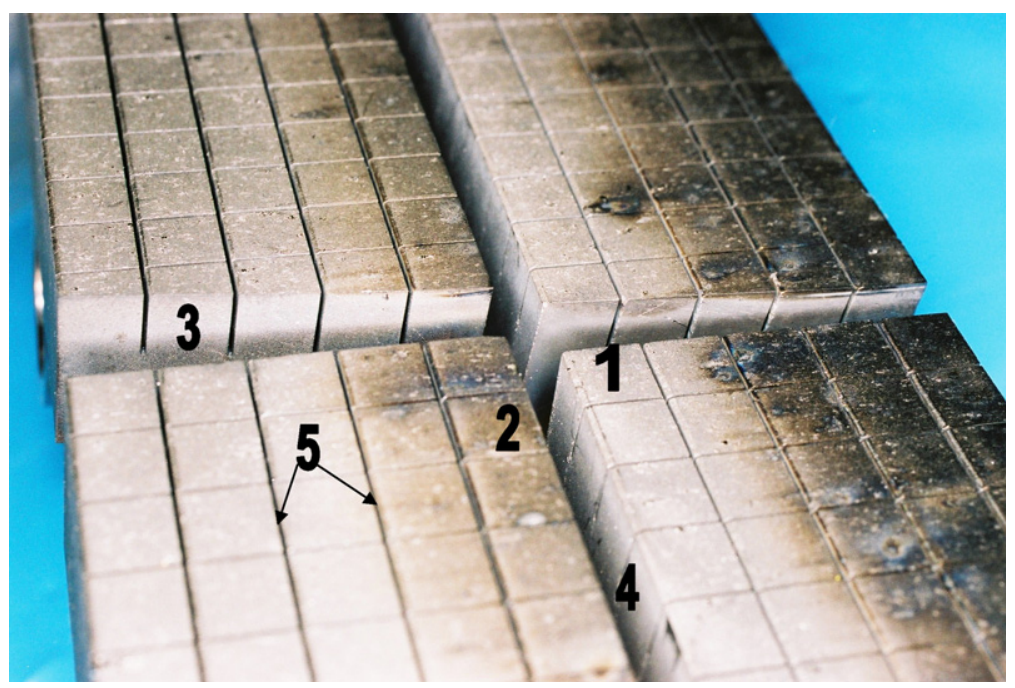

Fig. 1. Roof-shaped beryllium tiles of the Mk-I divertor: upper (1) and lower edge with an areas shadowed by the adjacent higher tiles (2); toroidal gap between tiles (3); poloidal gap between tiles (4); castellation groves (5).

surfaces and also the sides, i.e. on surfaces located in gaps between the tiles. The appearance of the plasma exposed CFC tiles with flaking co-deposits can be found in [9], whereas details regarding the elemental composition of plasma-facing surfaces of the divertor floor have been reported in [12]. Only a brief summary of the most important facts is given here. On many tiles the maximum inventory, exceeding $5 \times 10^{19} \mathrm{D}$ at $\mathrm{cm}^{-2}$, was detected in narrow ( $\sim 5 \mathrm{~mm}$ wide) deposition belts in the shadowed part of the tile. There were no areas with $\mathrm{D}$ concentrations lower than $5 \times 10^{17} \mathrm{~cm}^{-2}$. Assuming toroidal symmetry of the deposition pattern, the total inventory on the entire area of plasma-facing surfaces on the divertor floor $\left(11.1 \mathrm{~m}^{2}\right.$, with the projected gap surface area subtracted) could be assessed on the level of $8.9 \times 10^{23} \mathrm{D}$ atoms. This value should be treated as a lower limit, because the information depth for deuterium with NRA using a $2.5 \mathrm{MeV}^{3} \mathrm{He}^{+}$beam was limited to approximately $8-8.5 \mu \mathrm{m}$ whereas thicker co-deposits with very high deuterium content and the concentration ratio of deuterium-to-carbon, $\mathrm{D} / \mathrm{C} \sim 0.5$, were detected on some tiles.

Fig. 2 compares deuterium profiles on poloidally and toroidally-oriented side surfaces, illustrating the most important result that the deposition has occurred on the entire lateral surface. In the depth analysed by NRA no significant difference between the deposition in toroidal and poloidal gaps has been observed. The deuterium content varies in the gap but on average it is not less than $3 \times 10^{19}$

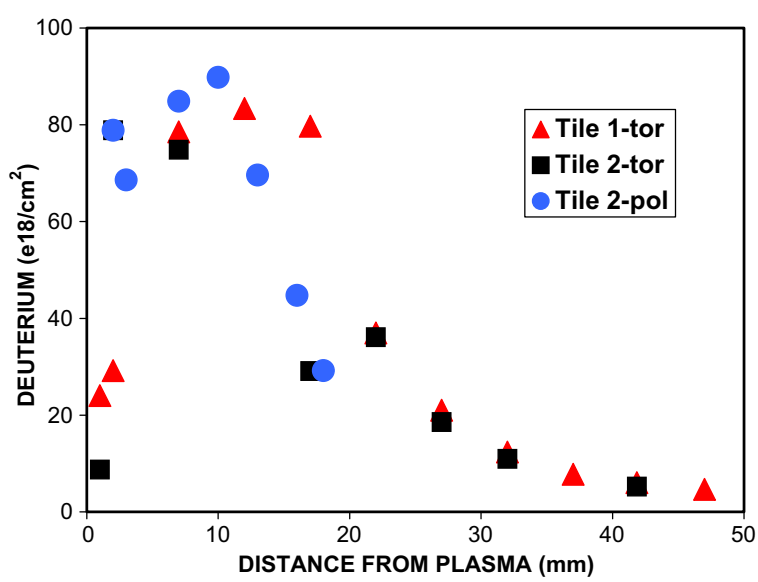

Fig. 2. Distribution of deuterium on side surfaces in the gaps between the Mk-I CFC tiles.

$\mathrm{D} \mathrm{cm}^{-2}$. On the same surfaces, the beryllium content is significantly smaller: up to $3 \times 10^{18} \mathrm{Be} \mathrm{cm}^{-2}$ in the region $1-3 \mathrm{~mm}$ from the plasma-facing edge and only $0.3-1.1 \times 10^{18} \mathrm{Be} \mathrm{cm}^{-2}$ in the lower part of the tile. One may therefore conclude that the fuel retention on the surfaces in gaps is associated with the transport of hydrocarbons and formation of carbon-rich co-deposits. A detailed surface analysis has been performed only for a limited number of tiles, but visual inspection of the whole poloidal set of divertor components confirms that the deposition pattern found on these isolated units was fairly typical of the entire divertor. Based on these results, the total integrated amount of fuel on side 
surfaces is assessed at the level of $15.9 \times 10^{23} \mathrm{D}$ atoms, i.e. nearly twice as high as found on plasma-facing surfaces. This has profound implications for the use of tile castellation in the presence of carbon in a machine with high duty cycle.

To assess whether the fuel stored in co-deposits migrates into the bulk, side surfaces of some CFC tiles were cleaved along the fibre planes. The deuterium on newly open surfaces $(1.0-1.5 \mathrm{~mm}$ beneath the original tile surface) was then determined with IBA using a $1.5 \mathrm{MeV}{ }^{3} \mathrm{He}^{+}$beam (information depth $4.5 \mu \mathrm{m})$. Measurements were performed ten months after tile retrieval from the torus. NRA spectra were recorded with a high dose of $3.75 \times$ $10^{15}{ }^{3} \mathrm{He}^{+}$ions to obtain decent statistics. Based on the analyses of ten points on the cleaved tile the $\mathrm{D}$ content on the surface was assessed in the range $0.35-5.3 \times 10^{15} \mathrm{~cm}^{-2}$ (in the $4.5 \mu \mathrm{m}$ thick layer) in comparison to the content exceeding $3 \times$ $10^{19} \mathrm{D} \mathrm{cm}^{-2}$ in the co-deposit on surfaces in gaps between the tiles. From these data one may conclude that deuterium migrates into the CFC used at JET (Concept I, Dunlop Ltd.), but this process is not considered to be crucial for the overall fuel inventory, which is mainly determined by the codeposition.

\subsection{Mk-I divertor with beryllium tiles}

The inspection of the castellated Be tiles (see also Fig. 1) has revealed three important features: (i) on the plasma-facing surfaces the deposition is particu- larly significant in the region shadowed by the adjacent tile; (ii) deposition on the side surfaces in gaps has occurred even at the bottom part of the divertor components, but no thick flaking co-deposits have been found; (iii) the castellation edges are still fairly sharp after long-term plasma exposure. Recent examination of the tiles from the inner divertor corner has permitted, for the first time, the assessment of material transport to the side surfaces and castellation grooves in Be. To make this study possible, a few 'teeth' of castellation were cut from a single tile, allowing comparison of the fuel content on two kinds of surfaces: located in the groove and in the gap between the tiles. Fig. 3 shows a detailed image of the tile in question with the fuel contents in various areas also indicated. The $\mathrm{D}$ concentration in the toroidal gap between the Be tiles decreases sharply by a factor of 3 at a distance of $6-8 \mathrm{~mm}$ from the plasma-facing surface. When compared to the carbon divertor (see Fig. 2) the total D content is over 10 times lower than on CFC. In the narrow castellated grooves the fuel content decreases by roughly an order of magnitude over a distance of $5 \mathrm{~mm}$, from $6 \times 10^{17} \mathrm{~cm}^{-2}$ near the plasma-facing edge to $8 \times 10^{16} \mathrm{~cm}^{-2}$ in the region deeper in the groove. The corresponding decay length, $\lambda$, is approximately $1.5 \mathrm{~mm}$. These concentrations are 70-100 times lower than those on plasma-facing surfaces [12] and in the gaps separating tiles.

From the analysis results compiled in [12], the integrated fuel content on the plasma-facing side of the inner corner Be tile could be estimated at

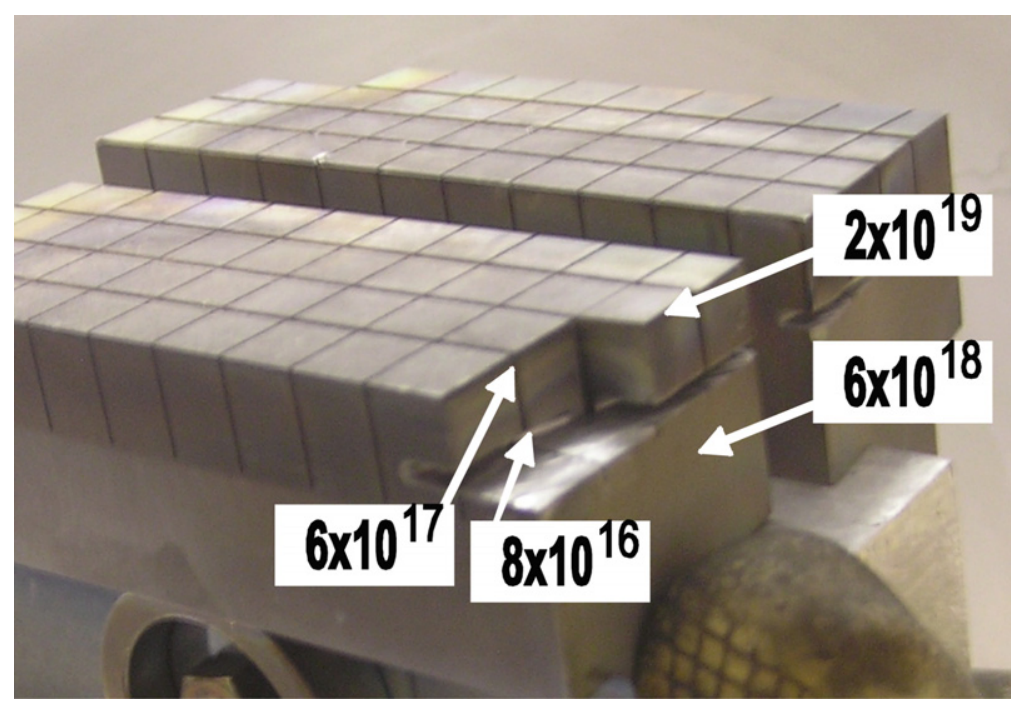

Fig. 3. Deuterium content on the side surfaces and in castellated grooves on the Mk-I beryllium tile. 
the level of $9.32 \times 10^{20} \mathrm{D}$ atoms $\left(C_{\text {surface }}\right)$. The recently determined integrated content in the castellation grooves of this tile is $\sim 0.22 \times 10^{19} \mathrm{D}$ atoms $\left(C_{\text {groove }}\right)$, whereas $3.05 \times 10^{20} \mathrm{D}$ atoms $\left(C_{\text {gap }}\right)$ are found on the surface located in the gap. One concludes that: (i) $C_{\text {groove }} / C_{\text {surface }}=0.024(2.4 \%)$ and (ii) $C_{\text {gap }} / C_{\text {surface }}=0.33(33 \%)$. The results clearly show that the fuel content in the shadowed regions of the $\mathrm{Be}$ divertor is distinctly lower than in the CFC structure.

The most important result is that deuterium on these tiles has always been detected together with carbon, indicating that co-deposition occurs in the gaps and in castellated grooves. Significant differences in deposition and fuel retention between the $\mathrm{CFC}$ and $\mathrm{Be}$ divertor cannot then be explained only by a three-times shorter operation time of Mk-I with Be tiles when compared to operation with the CFC components (see Section 2). All carbon found in the Be divertor must have originated from the erosion of the main chamber wall, so that the differences between the CFC and Be divertors may be attributed to the lack of the local carbon source in case of the Be divertor floor.

The fact that the retention in castellated grooves is found to be small in comparison to that on plasma-facing surfaces and in gaps between the tile demonstrates that the gap width plays an important role in determining the structure of the retention profile within the gaps. It is likely that at least some of the difference can be ascribed to the role of the electrostatic sheath at the tile surface. In a tokamak divertor, magnetic field line impact angles are extremely shallow: of order $1^{\circ}-3^{\circ}$ in the strike point regions during the JET campaigns of interest here. As a result, in addition to the ordinary Debye sheath (of thickness a few $\lambda_{\mathrm{D}} \sim 100 \mu \mathrm{m}$ ) at the tile surface, a quasi-neutral magnetic pre-sheath (or Chodura sheath [13]) is formed with a thickness of a few Larmor radii $\left(r_{\mathrm{L}}\right)$ :

$r_{\mathrm{L}} \approx\left(2 T_{\mathrm{e}} m_{\mathrm{i}} / e\right)^{1 / 2} / B$,

where $T_{\mathrm{e}}$ is the edge electron temperature, $m_{\mathrm{i}}$ the ion mass, $e$ is the elementary charge and $B$ the magnetic field strength. The extended length of the campaigns during which the tiles analysed here were exposed precludes specification of the precise plasma parameters in the divertor interaction area, but $T_{\mathrm{e}} \sim 10 \mathrm{eV}$ and $B \sim 3 \mathrm{~T}$ are typical giving $r_{\mathrm{L}} \sim 0.25 \mathrm{~mm}$ and Chodura sheath thickness of order $1 \mathrm{~mm}$. In addition, throughout campaigns with the $\mathrm{CFC}$ and $\mathrm{Be}$ divertors, the magnetic field and plasma current directions were maintained constant, so that magnetic field lines attack the divertor always from the same direction. A total (electrostatic and Chodura) sheath thickness of $\sim 1 \mathrm{~mm}$ exceeds the Be castellation groove width. As a consequence, the gaps are essentially shielded from the divertor plasma. The electrostatic field within the Chodura sheath acts to turn the supersonic ion flow from the direction parallel to the total field to the direction normal to the tile surface, thus maintaining the plasma surface interaction closely localized to the very tile edges. In the case of the larger tile gap (in the range 6-10 mm, Section 2), a sheath cannot bridge the gap and particles will penetrate slightly into the gap. Secondly, in the case of CFC tiles, the material chemically eroded from edges of the roof-shaped tiles could be transported down the tile gap. More detailed kinetic simulations are planned to investigate the matter further.

\section{Concluding remarks}

An important contribution of this work to tritium retention studies is the assessment of fuel inventory in castellated PFCs, i.e. in structures similar to those foreseen in ITER. Much more pronounced fuel accumulation is measured in the presence of the CFC structure than in case of the Be tiles. The essential conclusion derived from the comparison of deposition in the $\mathrm{CFC}$ and $\mathrm{Be}$ $\mathrm{Mk}-\mathrm{I}$ divertors is that fuel inventory in gaps and castellation grooves is associated with the deposition of carbon. The difference in fuel content in the gaps between the tiles (less in Be than in CFC structure) also indicates that co-deposition in the divertor may be attributed to two factors: (i) transport of species eroded from the main chamber wall; (ii) transport from the local carbon source in the divertor itself. The small inventory in the castellated grooves of beryllium tiles points to the probable influence of the gap width on the overall in-vessel fuel retention.

These results from JET as operated with a carbon wall and evaporated beryllium coating in the main chamber should not be immediately translated into conclusions and quantitative predictions regarding the material migration and fuel inventory in ITER. The planned material configuration in the divertor (W and CFC) and on the main chamber wall (Be) will be different than in any present-day device [14]. This will change both the scenario of material 
erosion and will influence fuel co-deposition and will be one of the main issues studied at JET when it is operated in the future with an ITER-like wall [15]. One may suggest on the basis of results presented here that in a machine with non-carbon walls in the main chamber, the material transport and resulting fuel inventory would be reduced. It is unreasonable to expect, however, that the deposition in the castellation grooves will be completely eliminated. The development of efficient techniques of fuel removal from all in-vessel components remains, therefore, crucial for the operation of a reactor-class device.

\section{Acknowledgements}

This work has been conducted under the European Fusion Development Agreement and is partly funded by EURATOM, the UK Engineering and Physical Sciences Research Council and the Swedish Research Council (VR) under the Contract VR-6212003-3170.

\section{References}

[1] P. Andrew et al., Fusion Eng. Des. 47 (1999) 243.

[2] C.H. Skinner et al., J. Nucl. Mater. 241-243 (1997) 214.

[3] J.P. Coad et al., J. Nucl. Mater. 290-293 (2001) 224.

[4] R.D. Penzhorn et al., J. Nucl. Mater. 288 (2001) 170.

[5] M. Rubel et al., Fusion Sci. Technol. 48 (2005) 569.

[6] W. Daenner et al., Fusion Eng. Des. 61\&62 (2002) 69.

[7] W.R. Wampler et al., J. Vac. Sci. Technol. A 6 (1988) 2111.

[8] C.H. Skinner, Nucl. Fusion 39 (1999) 271.

[9] M. Rubel et al., Phys. Scripta T111 (2004) 112.

[10] B. Emmoth, M. Rubel, E. Franconi, Nucl. Fusion 30 (1990) 1140 .

[11] M. Rubel et al., J. Nucl. Mater. 196-198 (1989) 285.

[12] J.P. Coad, M. Rubel, C.H. Wu, J. Nucl. Mater. 241-243 (1997) 408.

[13] R. Chodura, in: D.E. Post, R. Behrisch (Eds.), Physics of Plasma-Wall Interactions in Controlled Fusion, Plenum, New York, 1986, p. 99.

[14] R.A. Pitts et al., Plasma Phys. Control. Fus., 47 (2005) B303.

[15] G. Piazza et al., J. Nucl. Mater., these Proceedings, doi:10.1016/j.jnucmat.2007.04.039. 